\title{
Early Maladaptive Schemas in Development of Addictive Disorders as Parts of the Complex Addiction Jigsaw
}

\author{
Mark D Griffiths ${ }^{1, *}$ \\ ${ }^{1}$ International Gaming Research Unit, Psychology Division, Nottingham Trent University, Nottingham, UK \\ *Corresponding author: Mark D Griffiths, International Gaming Research Unit, Psychology Division, Nottingham Trent University, Burton Street, Nottingham, UK. Tel:+441-158482401, \\ E-mail: mark.griffiths@ntu.ac.uk
}

Received: December 10, 2013; Revised: February 21, 2014; Accepted: February 24, 2014

Keywords: Behavior; Addictive; Cognition Disorders

\section{Dear Editor,}

Addiction is a complex behavior (1). Acquisition, development, and maintenance of addictive behaviors are resulted from the interplay between many interacting factors (2) including the psychological and/or biological predispositions of the individual (e.g. genetic, attitudinal, belief and personality factors, unconscious motivations, etc.), the social environment in which the individual lives (i.e. situational characteristics), and the activity itself (i.e. structural characteristics) (3). This 'global' view of addiction highlights various integrative processes which interplay between these individual, situational and structural differences. Depending upon the type of addiction, these various overarching influences (i.e. individual, situational, and structural) can be subdivided even further. Addiction can be therefore conceptualized as a complicated and integrated jigsaw. The paper by Bakhshi Bojed and Nikmanesh (4) provided another piece in the 'addiction' jigsaw by demonstrating that specific individual differences (in this case, early maladaptive schemas developed in adolescence, i.e. disconnection/rejection; impaired autonomy and performance; impaired limits; other-directedness; over-vigilance/inhibition) show a significant positive correlation with addictive potential. Despite the highly significant positive correlational relationship, the study showed that even with three of the maladaptive schemas (i.e. disconnection/rejection; impaired autonomy and performance; other-directedness) added to the stepwise regression model, it still only explained $18 \%$ of the variance for addictive potential. Although these results appear to confirm the findings of other studies which have shown a positive correlation between early maladaptive schemas and drug addictions $(5,6)$, the study findings also revealed that vast majority of the addictive potential among adolescents is explained by many other influencing factors.
To paraphrase Burglass and Shaffer (7), two of the most important questions in the addiction field are "why do people become addicted to some things and not others?' and 'why some people become addicted and not others?' (of which, findings of Bakhshi Bojed and Nikmanesh contribute empirical evidence to the second question) (4). In a paper by Larkin and colleagues (8) examining the nature of addiction, responses to these two critical questions have been somewhat impeded because of two common misconceptions about such behavior: 1) Addiction somehow resides within a particular type of individual. 2) Addiction resides in a particular substance (in the case of chemical addictions) or activity (in the case of behavioral addictions). Put more simply, the misconception is that some individuals are already 'diseased,' or that some activities or substances cause the disease, or both. Findings of Bakhshi Bojed and Nikmanesh (like those investigating other factors such as influence of parents or the media) $(9,10)$ suggest that strategies are highly needed to prevent the addiction occurring in the first place. Majority of researchers have identified vulnerable groups with predispositions to developing addictions. As Bakhshi Bojed and Nikmanesh asserted, we need to create suitable preventive and therapeutic strategies based on the results of empirical work. Researchers should also be aware that addictions and dependencies are not just restricted to those who are involved with ingestion of a psychoactive substance. Research into maladaptive schemas could also be adapted for research into other addictions including behavioral addictions such as gambling, video game, exercise and shopping addictions (11). The complex systems model, as formulated by Griffiths and Larkin (1), corresponds well to the bio-psychosocial approach to addiction and can incorporate any addiction risk factor, including maladaptive schemas. Griffiths and Larkin have 
$(1,12)$ also argued for a complex systems model of addiction. 'Complex' for the obvious reason that addictive behavior is complex, and 'systems' based on the research of Davies (13), who proposed that alternative explanations for addictive behavior require "development of a 'system' within which drug usage is conceived as an activity carried out for positive reasons, by people who make individual decisions about their substance usage and who may take drugs competently as well as incompetently" (p.163). Based on the observations made by Polkinghorne, the paper by Griffiths and Larkin (1) argued that the complex systems model (which they contended that was a more theoretically flexible approach) goes "some way towards bridging the epistemological gap” (p. 101). Griffiths and Larkin also contended that the complex systems approach was descended from previous writings which take a multi-factorial approach in explaining addictive behaviors (14). It is also possible to consider the interplay between common and unique elements of any particular individual's situation and across the lifespan utilizing the complex systems model (including adolescence as highlighted in the paper by Bakhshi Bojed and Nikmanesh) (4). This includes many factors (i.e. physiological, social, psychological, and cultural) that may be specific to an individual person. Additionally, such an approach is all-inclusive and can include both the psychopharmacological properties of specific psychoactive substance addictions as well as the psychologically reinforcing properties of behavioral addictions such as pathological gambling. However, it such a model is not a return to locating the property of 'addictiveness' within a particular substance or within a particular activity, although those in the field need be aware of the effects (eg, physiological, psychological, social), that share commonalities with specific kinds of activities or substances, but not with others. It is clear that many research paradigms are insular and inadequate in explaining addiction. Development of maladaptive schemas on their own cannot and do not fully explain addictive behavior. Addictions are multi-faceted behaviors, cannot be explained by a single parsimonious theory, and can be strongly influenced by contextual factors (2). Research into (and treatment of) addictive behavior needs to be rooted within an interdisciplinary bio-psychosocial perspective, which eclectically incorporates the best theoretical approaches of contemporary biology, psychology, and sociology. According to Griffiths, a successful explanatory theory of addictive behaviors should contain a number of core components (1). More specifically, such a theory must "1) Synthesize pharmacological, cultural, situational and personality factors, 2) Account for the varying nature of addiction across cultures, individuals and time, 3) Account for commonalities between all addictions, and 4) Be faithful to lived human experience" (p.196). The paper by Bakhshi Bakhshi Bojed and Nikmanesh contributes further evidence for (and to) the multi-faceted nature of addiction (4).

\section{References}

1. Griffiths MD, Larkin M. Editorial conceptualizing addiction: the case for a "complex systems" account. Addict Res Theory. 2004;12(2):99-102.

2. Griffiths MD. A'components' model of addiction within a biopsychosocial framework. J Substance Use. 2005;10(4):191-7.

3. Parke J, Griffiths MD. The psychology of the fruit machine: The role of structural characteristics (revisited). Int J Ment Health Addict. 2006;4(2):151-79.

4. Bakhshi Bojed F, Nikmanesh Z. Role of early maladaptive schemas on addiction potential in youth. Int J High Risk Behav Addict. 2013;2(2):72-6.

5. Ball SA, Cecero JJ. Addicted patients with personality disorders: traits, schemas, and presenting problems. J Pers Disord. 2001;15(1):72-83.

6. Brotchie J, Meyer C, Copello A, Kidney R, Waller G. Cognitive representations in alcohol and opiate abuse: the role of core beliefs. Br J Clin Psychol. 2004;43(Pt 3):337-42.

7. Burglass ME, Shaffer H. Diagnosis in the addictions I: Conceptual problems. Adv Alcohol Subst Abuse. 1983;3(1-2):19-34.

8. Larkin M, Wood RTA, Griffiths MD. Towards addiction as relationship. Addict Res Theory. 2006;14(3):207-15.

9. Wakefield M, Flay B, Nichter M, Giovino G. Role of the media in influencing trajectories of youth smoking. Addiction. 2003;98 (Suppl1):79-103.

10. Wood RT, Griffiths MD. Adolescent lottery and scratchcard players: do their attitudes influence their gambling behaviour? JAdolesc. 2004;27(4):467-75.

11. Demetrovics Z, Griffiths MD. Behavioral addictions: Past, present and future. J Behav Addict. 2012;1(1):1-2

12. Larkin M, Griffiths MD. Response to Shaffer (1996): The Case for a 'Complex Systems' Conceptualisation of Addiction. J Gambl Stud. 1998;14(1):73-82.

13. Davies JB. The myth of addiction: an application of the psychological theory of attribution to illicit drug use. Harwood Academic Publishers, Chur; 1992.

14. Zinberg NE. Drug, set, and setting: The basis for controlled intoxicant use. Yale University Press New Haven; 1984. 